\title{
A Comparison and Analysis of Soft Computing Techniques for Content based Image Retrieval System
}

\author{
S. Manoharan \\ Associate Professor and Head \\ PG Department of Computer Science \\ Kongu Arts and Science College \\ Erode, Tamil Nadu, India - 638107.
}

\author{
S. Sathappan, PhD. \\ Associate Professor \\ Department of Computer Science \\ Erode Arts and Science College \\ Erode, Tamil Nadu, India - 638009.
}

\begin{abstract}
Content-based image retrieval has become one of the most active research areas in the past few years. In this paper various methodologies used in the research area of Content Based Image Retrieval methods using Soft Computing techniques are discussed. The comparison and analysis of various soft computing techniques like Fuzzy Logic (FL), Artificial Neural Network (ANN), Genetic Algorithm (GA) and Neuro-Fuzzy are performed. Soft Computing techniques are incorporated into Content-based image retrieval for obtaining more precise results. This is an open research area for the researchers in the field of Content-based image retrieval. This paper covers various Soft Computing techniques for Content Based Image Retrieval Systems (CBIR), the parameters used for experimental evaluation of the systems and the analysis of these techniques on the basis of their results.
\end{abstract}

\section{General Terms}

Content Based Image Retrieval Techniques, Soft Computing Techniques.

\section{Keywords}

Content-based image retrieval, Soft Computing Techniques, Artificial Neural Network, Fuzzy Logic, Genetic Algorithm, Neuro-Fuzzy.

\section{INTRODUCTION}

Since 1990s, the demand for information indexing and retrieval is increased because of rapid growth of available multimedia contents, that's why efforts have been done on text extraction in images and videos. Then the total Collection of images in the web are growing larger and becoming more diverse. Retrieving images from such large collections is a challenging problem in front of us. To organize and classify such large amount of images is time consuming task. Therefore it is required to design a system to organize and classify the images in a database, so that the images can be retrieve fast with little amount of time. To implement this idea, the CBIR system is introduced. It is an automated system that searches query image in an image database and retrieving the relevant images of using similarity measure between it and every image in the image data base. It can simplify many tasks in many application areas such as biomedicine, forensics, artificial intelligence, military, education, web image searching and etc. [1]
In recent years, there has been a growing interest in developing effective methods for content based image clustering and retrieval. This interest has been motivated by the need to efficiently manage large image databases and efficiently run image retrieval to get the best results without exhaustively searching the global database each time. This leads to huge savings in time and money, especially in fields where the bulk of working databases are image files or any kind of media whose contents cannot be described by simple keywords or short texts. With image retrieval system such as Content Based Image Retrieval, this limitation will not exist and system will retrieve the images as per the human can perceive, and too much responsibility on the end-user, problem of abstract needs, the queries that cannot be described at all, but tap into the visual features of images.[2]

\section{CONTENT BASED IMAGE RETRIEVAL SYSTEMS (CBIR)}

The overall structure of a typical image retrieval system is shown in figure 1. It consists of images, feature extraction and retrieval process. First the images are obtained from different sources. One of the images from the existing source is considered as query image and remaining images forms an image database. The feature extraction process is applied on images in the database and the query image and the feature vector is obtained. The search \& retrieval process search the query image matched with images in the database. Finally images are received and arranged according to their matching score. The Content-based query processing lies at the heart of all CBIR systems. Processing of query (image or graphics) involves extraction of visual features and/or Segmentation and search in the visual feature space for similar images. An appropriate feature representation and a similarity measure to rank pictures, given a query, are essential here. Processing text-based queries involves keyword matching using simple set-theoretic operations, and therefore a response can be generated very quickly. However, in very large systems working with millions of pictures and keywords, efficient indexing methods may be required. Indexing of text has been studied in database research for decades now. Efficient indexing is critical to the building and functioning of very large text based databases and search engines. Research on efficient ways to index images by content has been largely overshadowed by research on efficient visual representation and similarity measures. Most of the methods used for visual indexing are adopted from text-indexing research [2].

The low level features of content based image retrieval like color feature; Texture feature, shape feature and layout feature 
are widely used in the image feature extraction. These feature extraction methods are playing vital role in the image retrieval. The various similarity measures are also used during the process of Content Based Image Retrieval [22].

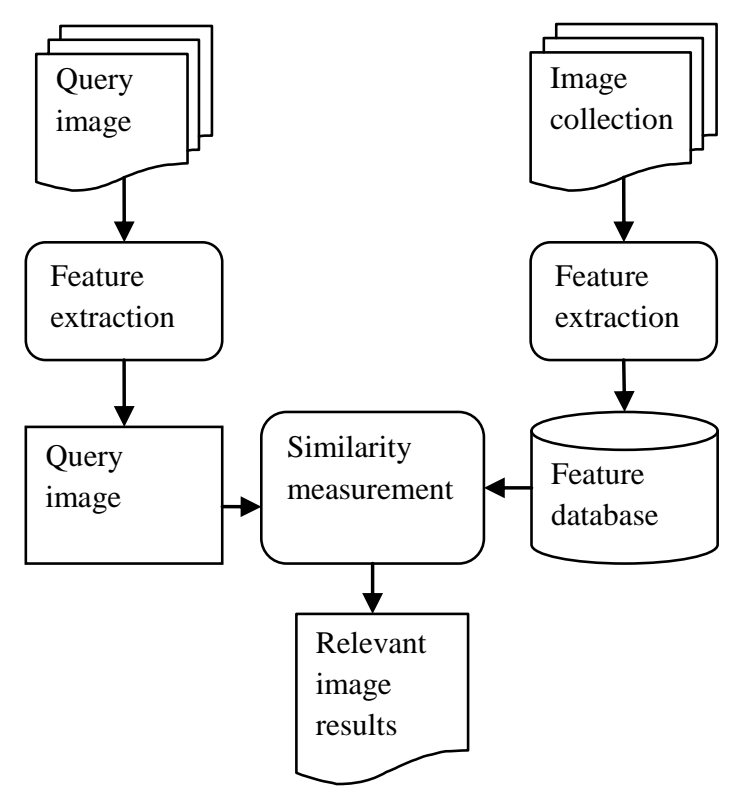

Figure 1: Content Based Image Retrieval System

\section{SOFT COMPUTING TECHNIQUES FOR CBIR}

Soft computing consists of set of technologies like Artificial Neural Network (ANN), Fuzzy logic (FL), Genetic Algorithm (GA), Machine learning and probabilistic reasoning. Due to their strong learning, cognitive ability and good tolerance of uncertainty and imprecision, soft computing techniques have found wide applications. Image processing and retrieval is one of such applications. The aim of this paper is to analysis and compares some soft computing techniques for Content Based Image Retrieval System (CBIR) and provides their retrieval efficiency based on precision and recall rate.

\section{Number of Retrieved images relevant to the query Image}

\section{Precision $=$ \\ Total number of images retrieved}

Number of Retrieved images relevant to the query Image

Recall $=$ Total number of relevant images in the database

\subsection{Artificial Neural Network (ANN) Technique for CBIR}

The Neural Network-based Image Retrieval (NNIR) which is the human-computer interaction system model of contentbased mage retrieval using the Radial Basis Function (RBF) network [3]. The RBF network is chosen in this system, because BP (Back Propagation) requires a large set of classified training data and RBF network is more appropriate to reflect the subsequent feedback of the user [4]. Initially, the RBF network itself contains off-line trained weight matrices to combine heterogeneous features and reflects the demand of the user on-line by iteration. The input to the RBF network is the set of metric values of each pair of images and the output is a number between 0 and 1 signifying similarity of images based on various input features. Using a set of training data, NNIR assigns weights to the features and refines those using the feedback from users. NNIR offers a new method of combining low level image features and reflecting the feedback of the user. Using combined, rather than individual, features is especially efficient for generic image databases, for which no single feature is outstanding. Experimental evaluation will demonstrate that the proposed model can achieve both efficiency and flexibility on content-based image retrieval using heterogeneous features of images [21].

The Neural Network-based Image Retrieval system is a human-computer interaction approach to CBIR. Unlike the linear combining approach, where the user has to precisely decompose his information need into different feature representations and precisely specify all of the weights associated with them, the proposed NNIR approach allows the user to submit a coarse initial query, and continuously refine his information need via user-friendly relevance feed-back. Moreover, this approach need not build the index structure using low-level features. By applying the Radial Basis Function network, this approach determines directly nonlinear relationships between features so that more accurate similarity comparison between images can be supported. The experimental results show that the proposed approach has $94.35 \%$ recall and $81.45 \%$ precision rate, which is the superior retrieval performance to the existing linear combining method, the rank-based method, and the BP-based method. Although the proposed retrieval model is for CBIR, it can easily be expanded to handle other media types, such as video and audio [19][20].

\subsection{Fuzzy Logic (FL) Technique for CBIR}

Fuzzy logic offers a good solution for posing a query in terms of natural language based on the various features of an image. Fuzzy logic has been extensively used at various stages of image retrieval such as region groupings within the images as a feature extraction technique, for measuring the similarity between the target image and the images in the database. Fuzzy logic is proposed for the computation of fuzzy colour histogram as well as posing the queries in CBIR. This paper reviews the prominent CBIR systems along with fuzzy logic based techniques and proposes a technique based on fuzzy logic and neural networks for retrieving the images using natural language query for colour and texture features fuzzy logic based similarity is proposed between the two images [5]. The weights are assigned to fuzzy colour content during the calculation of similarity between the two images. Assigning the weights for each fuzzy content term: very small $\rightarrow 0.1$, small $\rightarrow 0.25$, rather small $\rightarrow 0.4$, medium $\rightarrow 0.55$, rather large $\rightarrow 0.70$, large $\rightarrow 0.85$, very large-> 1 . A fuzzy intersection is the lower membership in both sets of each element. The fuzzy intersection of two fuzzy sets $A$ and $B$ on universe of discourse $\mathrm{X}: \mathrm{m} A C ̧ B(x)=\min [\mathrm{m} A(x), \mathrm{m} B(x)]=$ $\mathrm{m} A(x) \quad C \mathrm{~m} B(x)$, where $x \hat{\mathrm{I} X}$ The union is the largest membership value of the element in either set. The fuzzy operation for forming the union of two fuzzy sets $\mathrm{A}$ and $\mathrm{B}$ on universe $\mathrm{X}$ can be given as: $\mathrm{mAÈB}(\mathrm{x})=\max [\mathrm{mA}(\mathrm{x}), \mathrm{mB}(\mathrm{x})]$ $=\mathrm{mA}(\mathrm{x}) \mathrm{E} \mathrm{mB}(\mathrm{x})$, where $\mathrm{xIX}$. Finally the fuzzy similarity distance is $\mathrm{mAÈB}(\mathrm{x})-\mathrm{m} A C ̧ B(x)$. 
Based on the fuzzy image features and similarity measure, experiments were conducted on image data base. Fuzzy logic has been used extensively in various areas to improve the performance of the system and to achieve better results in different applications. This paper has proposed and investigated a novel approach based on natural language fuzzy logic queries, fuzzy mapping of image database and fuzzy similarity distance for retrieving the images based on their contents. The performance of the system was evaluated on real fuzzy logic for the interpretation of the texture queries for content-based image retrieval is proposed. User can pose a textual description or visual examples to find the desired image from the database. This technique simplifies the similarity between the query image and the images in the database and works well for CBIR [6].

\subsection{Genetic Algorithm (GA) Technique for CBIR}

CBIR has been an active area of research for long due to its applications in various fields like satellite imaging, medicine etc [7]. In order to determine which images must be retrieved, content representations of all stored images are compared with the representation extracted from the query image. The most important considerations in the design and implementation of CBIR systems are: image feature extraction, features representation, features matching, database organizing and querying mechanisms [7]. This paper broadly classifies the CBIR process as feature extraction and GA-based similarity measure [23].

An efficient CBIR system based on GA for retrieving relevant images from image database, a query image is given. First, the system extracted the features like texture, color and shape from the image. Then, with the aid of GA-based similarity measure, images that are similar to the query image have been retrieved effectively. The proposed CBIR technique has been evaluated by querying different images. The implementation results have shown that the proposed technique effectively retrieves the relevant images from the database. This could be visualized from the precision-recall determined from the retrieval results [8]. The proposed CBIR based on GA have the benefit of the shape feature in addition to other features, and the similarity measure using GA. The extracted images are $70 \%$ similar to the given query image.

\subsection{Neuro-Fuzzy Based Clustering Technique for CBIR}

The process of grouping a set of objects or patterns into classes of similar objects is called clustering. Clustering is a process that organizes a data set into a number of groups (clusters) such that patterns within a cluster are more similar to each other than patterns belonging to different clusters. In other words, clustering is an important technique for discovering the inherent structure in any given pattern set. It has been applied across many disciplines including engineering, statistics, psychology, sociology, astronomy, biology, business, medicine to name a few [9][10]. There exist two categories for clustering task: hard and soft clustering. In hard clustering, each data object is assigned to exactly one cluster, while soft clustering is more desirable to let a data object be assigned several clusters partially. Hence, the soft clustering is also called neuro-fuzzy clustering [14][15]. The goal of this paper is to provide methodology that concentrates on retrieving of images, defined by variety of clusters, in order to find the particular clusters. Color-texture based image retrieval methods with use of neuro-fuzzy system were shown in figure 2. From the initial returned images, users can select different clusters and retrieve from the database [18].

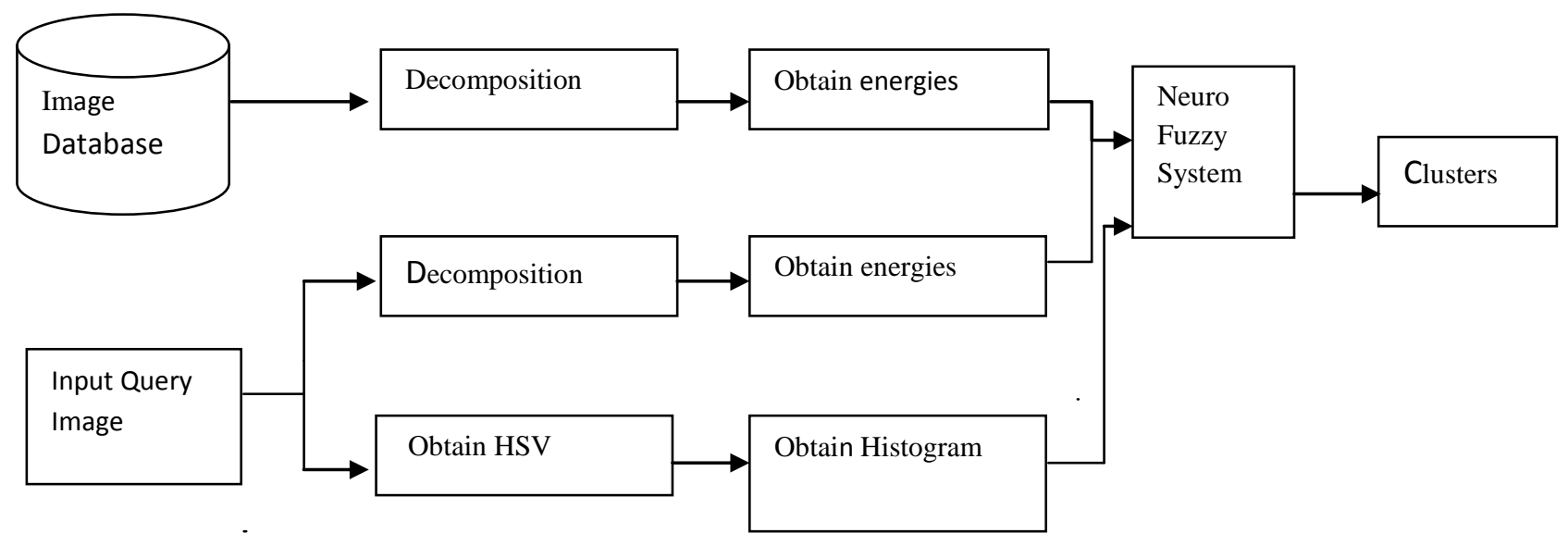

Figure 2: Neuro-Fuzzy Based Clustering Technique for CBIR

\subsection{Neural Network and Genetic Algorithm based Technique for CBIR}

An approach is described to content-based retrieval of medical images from a database provide a preliminary demonstration of a new approach as applied to retrieval of digital mammograms. In the medical-imaging context, the ultimate aim of Content Based Image Retrieval (CBIR) is to provide radiologists with a diagnostic aid in the form of display of relevant past cases, along with proven pathology and other suitable information. This paper proposes a new hybrid approach to content-based image retrieval. Contrary to the single feature vector approach which tries to retrieve similar images in one step, this method uses a two-step approach to retrieval. In the first step, the use of a neural network called Self Organizing Map (SOM) for clustering the images with respect to their basic characteristics were proposed [11]. In the second step, the GA based search will be made on a sub 
set of images which were having some basic characteristics of the input query image. Then new Hybrid approach was applied to a database of high resolution mammogram images and show that this method radically improves the retrieval precision over the single feature vector approach. To determine whether this CBIR system is helpful to physicians, an evaluation trial with five radiologists were conducted. The results show that the proposed system using genetic algorithms and neural network retrieval doubled the doctors' diagnostic accuracy. Moreover, this method is faster and has higher retrieval accuracy compared to the single stage methods [12]. Figure 3 shows the Neural Network and Genetic Algorithm based Technique for CBIR [17][24].

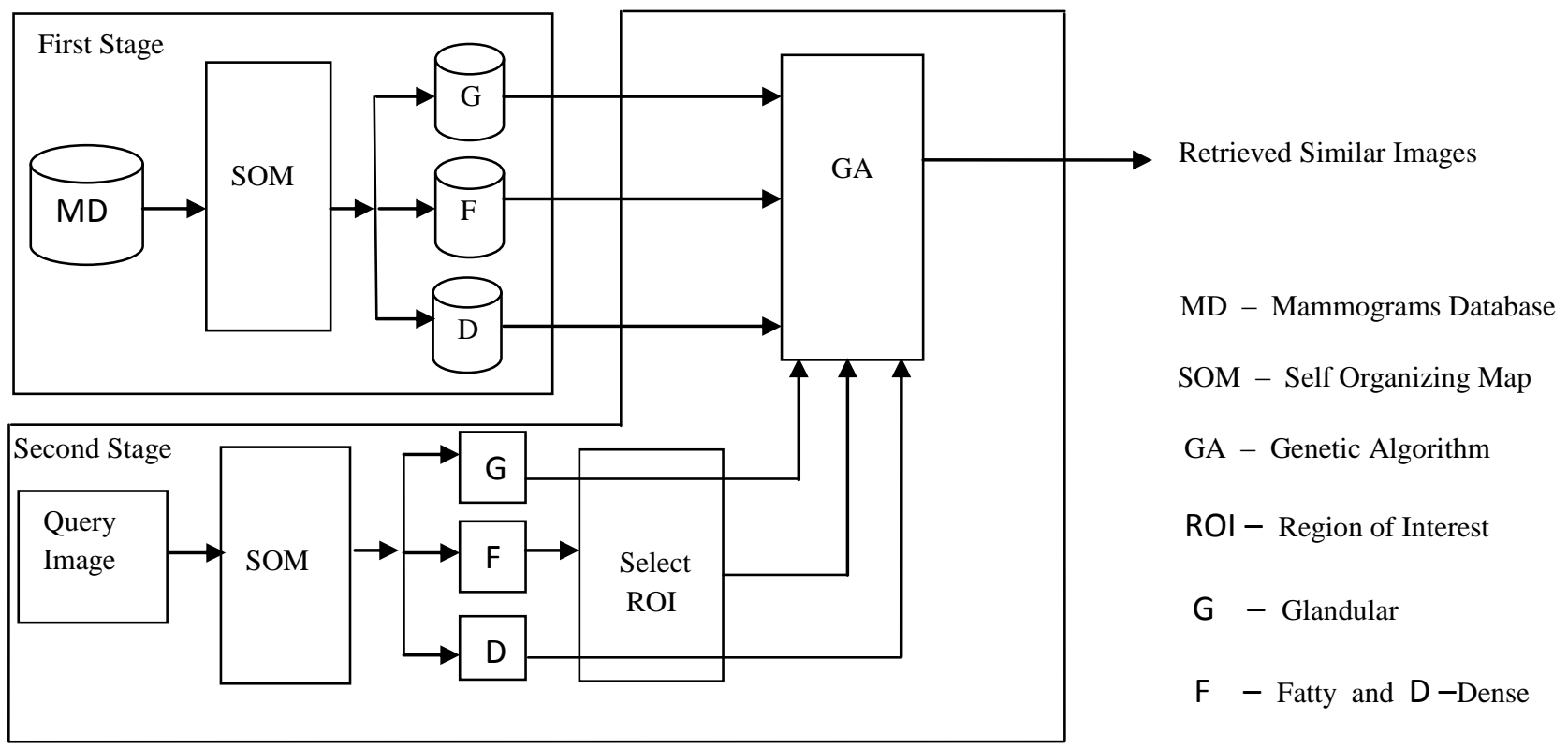

Figure 3: Neural Network and Genetic Algorithm based Technique for CBIR

\section{COMPARISIONS AND ANALYSIS OF FIVE SOFT COMPUTING TECHNIQUES FOR CBIR}

The various soft computing techniques for Content Based Image Retrieval were discussed in this paper. The performance and accuracy of the various techniques like Artificial Neural Network (ANN), Fuzzy Logic (FL), and Genetic Algorithm (GA) were also individually determined. Then the results of the hybrid soft computing techniques namely Neuro-Fuzzy Based Clustering Technique (ANN \& FL) for CBIR and Neural Network and Genetic Algorithm based Technique (ANN \& GA) for CBIR were also discussed. The performance and accuracy in terms of precision and recall has been improved in the hybrid soft computing techniques than they performed individually [14]. The following tables show the precision and recall rate for various soft computing techniques for CBIR. The following tables show the precision and recall rate for various soft computing techniques for CBIR. The table 1 gives the information about average precision rates of various soft computing techniques for the input query images ranging from 10 to 50 . The table 2 gives the information about average recall rates of various soft computing techniques for the input query images ranging from 10 to 50 . The experiments for all the above five methods were done in Mat lab and the results of the respective methods were also retrieved. The retrieved results of the various soft computing techniques of CBIR were analyzed and compared with each other methods. The soft computing techniques outperform the traditional CBIR methods in terms of precision and recall rate [16][25].
Table 1: Average Precision Rate for various Soft Computing Techniques

\begin{tabular}{|c|c|c|c|c|c|}
\hline \multirow{2}{*}{$\begin{array}{c}\text { Appro } \\
\text { ach }\end{array}$} & \multicolumn{5}{|c|}{ Average Precision Rate } \\
\cline { 2 - 6 } & 10 & 20 & 30 & 40 & 50 \\
\hline ANN & 0.763 & 0.764 & 0.764 & 0.764 & 0.766 \\
\hline FL & 0.719 & 0.718 & 0.714 & 0.713 & 0.719 \\
\hline GA & 0.711 & 0.729 & 0.729 & 0.729 & 0.729 \\
\hline $\begin{array}{c}\text { ANN } \\
\text { \& FL }\end{array}$ & 0.980 & 0.980 & 0.980 & 0.980 & 0.980 \\
\hline $\begin{array}{c}\text { ANN } \\
\text { \& GA }\end{array}$ & 0.960 & 0.960 & 0.960 & 0.960 & 0.960 \\
\hline
\end{tabular}


Table 2: Average Recall Rate for various Soft Computing Techniques

\begin{tabular}{|c|c|c|c|c|c|}
\hline \multirow{2}{*}{$\begin{array}{c}\text { Appr } \\
\text { oach }\end{array}$} & \multicolumn{5}{|c|}{ Average Recall Rate } \\
\cline { 2 - 6 } & 10 & 20 & 30 & 40 & 50 \\
\hline ANN & 0.771 & 0.772 & 0.773 & 0.774 & 0.786 \\
\hline FL & 0.787 & 0.780 & 0.775 & 0.781 & 0.789 \\
\hline GA & 0.691 & 0.699 & 0.698 & 0.699 & 0.699 \\
\hline $\begin{array}{c}\text { ANN } \\
\& \text { FL }\end{array}$ & 0.980 & 0.980 & 0.980 & 0.980 & 0.980 \\
\hline $\begin{array}{c}\text { ANN } \\
\& G A\end{array}$ & 0.960 & 0.960 & 0.960 & 0.960 & 0.960 \\
\hline
\end{tabular}

The number of query images versus the average precision rate of the five different soft computing techniques for CBIR is shown in figure 4 . The number of query images versus the average Recall rate of the five different soft computing techniques for CBIR is shown in figure 5. The performance and accuracy in terms of speed of retrieval and retrieval of required query image are more appropriate by Neuro-Fuzzy based CBIR technique [26].

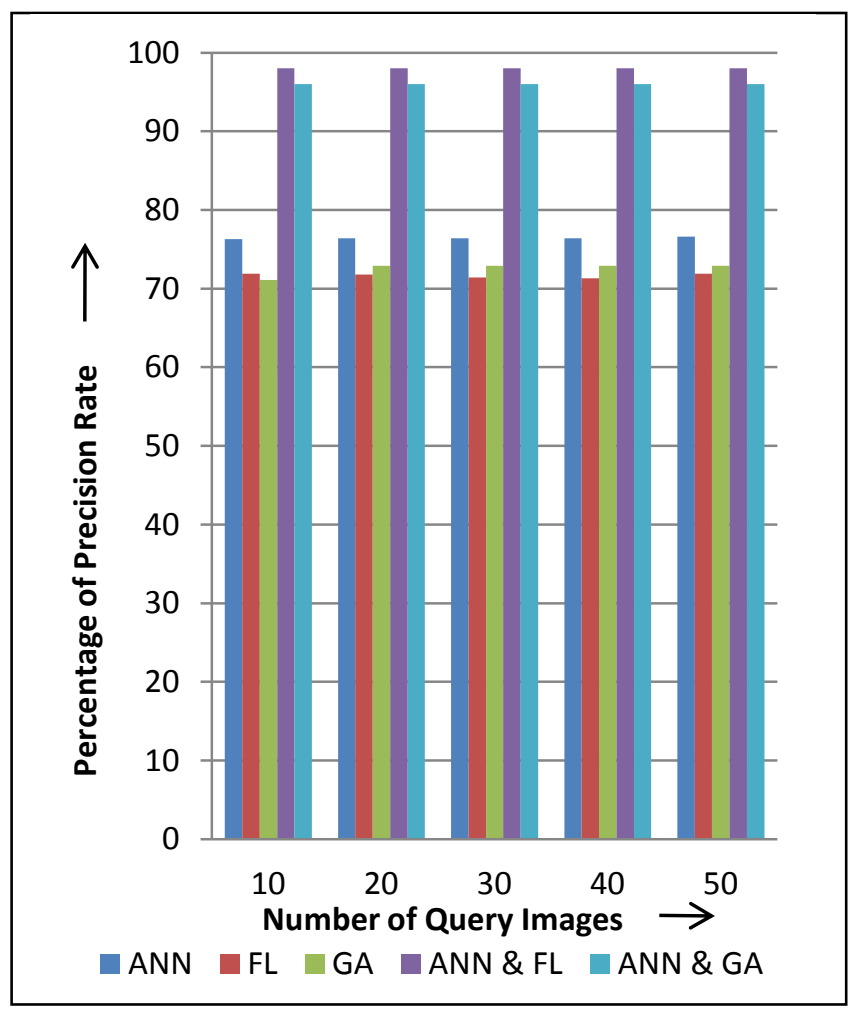

Figure 4: Average Precision Rate for various Soft Computing Techniques

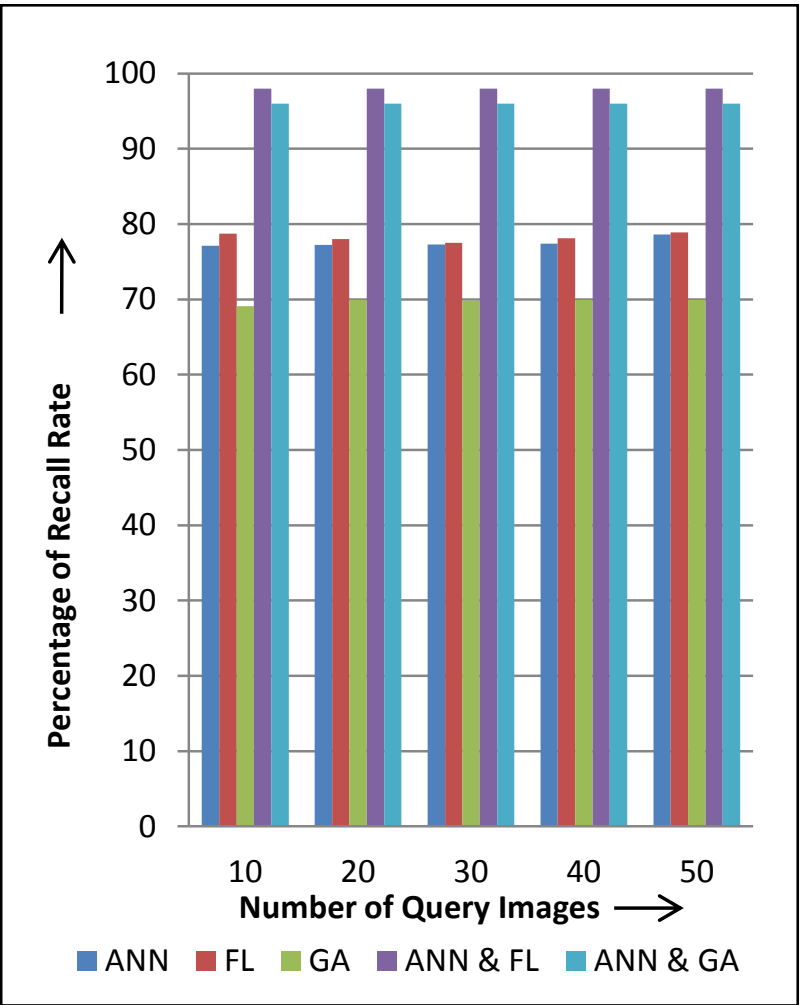

Figure 5: Average Recall Rate for various Soft Computing Techniques

\section{CONCLUSION}

This paper has analyzed and compared five soft computing CBIR methods. Method 1 employs the Artificial Neural Network by using the Radial Basis Function (RBF) network for CBIR. Method 2 utilizes the Fuzzy Logic technique by incorporating fuzzy colour histogram for CBIR. Method 3 uses Genetic Algorithm by applying GA-based similarity measure for CBIR. Method 4 employs Neuro -Fuzzy Based Clustering Technique for CBIR and Method 5 utilizes Neural Network and Genetic Algorithm based Technique for CBIR. Experimental results demonstrate that the precision and recall rates of these five methods and the Neuro -Fuzzy Based Clustering Technique for CBIR outperform the other existing soft computing methods. The ANN with GA approach reserves the second place in the performance and accuracy of image retrieval system. The remaining soft computing techniques like ANN, FL and GA have less accuracy and performance in image retrieval than the hybrid soft computing techniques like neuro-fuzzy and ANN with GA. In the future research, the existing neuro-fuzzy approach for image retrieval can be applied in the field of medicine to get more appropriate images from the image database and also the authors shall search for better feature combination and employ more databases to evaluate the above five methods. In addition, authors shall employ these five methods for video retrieval.

\section{ACKNOWLEDGEMENTS}

The authors of this paper would like to express their sincere thanks to the Management, Principal of their respective institutions and the faculty members of their respective departments who have contributed kind support to publish this paper. In addition, the authors also wish to convey their great 
gratitude to their family members who have rendered kind support to bring this research paper for the publication. The authors also express their sincere thanks to anonymous reviewer of IJCA for reviewing this work and providing necessary comments.

\section{REFERENCES}

[1] Bird, C.L.; P.J. Elliott, Griffiths (1996). User interfaces for content-based image retrieval.

[2] Datta, Ritendra; Dhiraj Joshi, Jia Li, James Z. Wang (2008). "Image Retrieval: Ideas, Influences, and Trends of the New Age". ACM Computing Surveys 40 (2): 1-60.

[3] D.S. Broomhead and D. Lowe, "Multivariable functional interpolation and adaptive networks," Complex Systems, vol.2, no.3, pp.321-355, 1988.

[4] M. Wood, N. Campbell, and B. Thomas, "Iterative refinement by relevance feedback in content-based digital image retrieval," Proc. ACM Multimedia 98, pp.13-20, Bristol, UK, Sept. 1998.

[5] Sticker, M., and Dimai, A., (1997). Spectral Covariance and Fuzzy Regions for Image Indexing, Machine Vision Application, Vol. 10, pp. 66-73.

[6] Vertan, C., and Boujemaa, N., (2000), Embedding Fuzzy Logic for Image Retrieval, 19th International Conference of the North American, pp. 85-89.

[7] Carneiro, G., Chan, B., Moreno, P., and Vasconcelos, N., "Supervised Learning of Semantic Classes for Image Annotation and Retrieval," IEEE Transactions on Pattern Analysis and Machine Intelligence, vol. 29, no. 3, pp. 394-410, 2007.

[8] Syam, B., and Rao, S., "Integrating Contourlet Features with Texture, Color and Spatial Features for Effective Image Retrieval", ICIME, vol.3, 2010.

[9] Trojacanec, Dimitrovski and Loskovska, "Content based image retrieval in medical applications: An improvement of the two-level architecture" in proceedings of IEEE EUROCON, pp.118-121, May 2009.

[10] Tsai, Y., "Salient Points Reduction for Content- Based Image Retrieval," World Academy of Science, Engineering and Technology, vol. 49, 2009.

[11] H.C.Lin,C.Y.Chiu, and S.N.Yang, "Finding textures by descriptions,visual examples and relevance feedbacks," IEEE Pacific. Rim Conference on Multimedia, pp. 308315.2001

[12] B.S. Manjunath and W.Y. Ma, "Texture Features for Browsing and Retrieval of image Data", IEEE Trans. On Pattern Analysis and Machine Intelligence; Vol 18, No. 8, pp. 837-842, 1996.

[13] M.Kakare,B.N.Chatterji, and P.K. Biswas, "Wavelet transform based texture features for content based image retrieval,"in Proc.9th Nat.Conf.on Communications(NCC 2003), Chennai,India,pp.443-447.Feb.2000.
[14] P. Muneesawang, L. Guan, "Automatic machine interactions for content-based image retrieval using a self organizing tree map architecture", IEEE Trans.on neural networks ,vol.13.,no.4,pp.821-834,July 2002.

[15] Jose, J. and T.P. Mythili, 2007. A model based tumor detection in brain MRI using genetic algorithm based image warping and template matching techniques. Proceedings of the International Conference on Information Systems and Technology, Dec. 14-15, MES College of Engineering, Kuttippuram, Kerala, India, pp: 120-125.

[16] M.Kakare,B.N.Chatterji, and P.K. Biswas, "Wavelet transform based texture features for content based image retrieval,"in Proc.9th Nat.Conf.on Communications(NCC 2003),Chennai,India,pp.443-447.Feb.2000.

[17] P. Muneesawang, L. Guan, "Automatic machine interactions for content-based image retrieval using a self organizing tree map architecture", IEEE Trans.on neural networks ,vol.13.,no.4,pp.821-834,July 2002.

[18] Jose, J. and T.P. Mythili, 2007. A model based tumor detection in brain MRI using genetic algorithm based image warping and template matching techniques. Proceedings of the International Conference on Information Systems and Technology, Dec. 14-15, MES College of Engineering, Kuttippuram, Kerala, India, pp: 120-125.

[19] J. Vogel and B. Schiele, "Performance evaluation and optimization for content-based image retrieval," Pattern Recognition, vol. 39, pp. 897-909, 2006.

[20] X. Y. Wang, Y. J. Yu, and H. Y. Yang, "An effective image retrieval scheme using color, texture and shape features," Computer Standards \& Interfaces, vol. 33, pp. 59-68, 2011.

[21] Y. P. Wang, K. T. Lee, and K. Toraichi, "Multiscale curvature-based shape representation using B-spline wavelets," IEEE Transactions on Image Processing, vol. 8, pp.1586-1592, 1999.

[22] C.-Y. Wee and R. Paramesran, "On the computational aspects of Zernike moments," Image and Vision Computing, vol. 25, pp. 967-980, 2007.

[23] C.-H. Wei, Y. Li, W.-Y. Chau, and C.-T. Li, “Trademark image retrieval using synthetic features for describing global shape and interior structure," Pattern Recognition, vol. 42, pp. 386-394, 2009.

[24] W.-T. Wong and S.-H. Hsu, "Application of SVM and ANN for image retrieval," European Journal of Operational Research, vol. 173, pp. 938-950, 2006.

[25] D. Zhang and G. Lu, "Review of shape representation and description techniques," Pattern Recognition, vol. 37, pp. 1-19, 2004.

[26] D. Zhang and G. Lu, "A comparative study of curvature scale space and Fourier descriptors for shape-based image retrieval," Journal of Visual Communication and Image Representation, vol. 14, pp. 39-57, 2003. 